\title{
Traduire
}

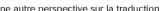

Revue française de la traduction

$218 \mid 2008$

De traduction en retraduction

\section{Le problème de la nuance en traduction pragmatique}

\section{Nicolas Froeliger}

\section{Q OpenEdition}

1 Journals

Édition électronique

URL : http://journals.openedition.org/traduire/901

DOI : $10.4000 /$ traduire.901

ISSN : 2272-9992

Éditeur

Société française des traducteurs

Édition imprimée

Date de publication : 15 juin 2008

Pagination : 77-93

ISSN : 0395-773X

Référence électronique

Nicolas Froeliger, «Le problème de la nuance en traduction pragmatique », Traduire [En ligne], 218|

2008, mis en ligne le 15 juin 2008, consulté le 21 décembre 2020. URL : http://

journals.openedition.org/traduire/901 ; DOI : https://doi.org/10.4000/traduire.901 


\title{
Le problème de la nuance en traduction pragmatique*
}

\author{
Nicolas Froeliger
}

Le Talmud affirmait déjà : "L'omission ou l'addition d'une lettre pourrait conduire à la destruction du monde." Certains illuminés allaient jusqu’à supposer que c'était une erreur de transcription, même infime, commise par le scribe à qui Dieu dictait le texte sacré, qui était à l'origine des ténèbres et des soubresauts du monde. (Steiner, 1978, p. 68)

Traduire, nous le savons tous, c'est, entre autres choses, bâtir une représentation du problème posé par le texte d'origine, et jeter sur le papier - via un clavier, un écran et une imprimante - une représentation équivalente en langue d'arrivée : comprendre, réexprimer. Cette démarche suppose que le traducteur soit capable de se représenter lui-même, pour déterminer quelle posture adopter face à son texte et pouvoir, dans un second temps, se projeter dans le personnage auquel il s'adresse. C'est une telle représentation que je voudrais proposer. Pour moi, le traducteur est géomètre, et son principe est la mesure. En toutes choses.

Qu'est-ce que la géométrie, pour commencer ? Les définitions abondent. De manière empirique, c'est tout ce que l'on peut faire avec une règle et un compas $(1)$. Dans la conduite de la réflexion, on pourra aussi retenir la définition de René Descartes : "Art de raisonner juste sur des figures fausses. " Et l'on se souviendra que la géométrie est aussi la transposition dans l'espace des relations algébriques (tout ce qu'on peut faire avec des chiffres). Il s'agit donc d'exprimer quelque chose par autre chose : c'est finalement un art de la traduction.

* Cette communication a été présentée par l'auteur à l'occasion de la 6e Journée de la traduction professionnelle (Lyon, 4 avril 2008), ndlr.

(1) Voir Warusfel, 1961, p. 85. 
Nous allons donc tenter d'explorer cette analogie afin de montrer en quoi la représentation du traducteur en géomètre permet de penser son activité et, idéalement, de servir de modèle pour produire des textes plus efficaces. Ce qui est tout de même le but de toute traductologie appliquée. Il nous semble en effet souhaitable de contribuer, modestement, à combler une des failles des études en traduction, décrite par Jean-René Ladmiral en ces termes : "Il y a induction de la théorie à partir de la pratique, mais le retour déductifne se fait pas(2). " Car la justesse de ce constat n'oblige pas à se satisfaire de cet état de choses...

Ce portrait du traducteur trouve en particulier son utilité face aux nuances. J'aimerais envisager celui-ci sous deux aspects principaux, accessibles aux outils du géomètre : l'importance que revêt généralement le respect de ces nuances (la règle), et la nécessité, parfois, de ne pas les respecter (le compas). Nous procéderons ainsi par ordre de complexité. Et je me servirai d'exemples, tirés aussi bien de la vie réelle que de mémoires d'étudiants du master professionnel dont je m'occupe (3).

\section{La règle et le compas}

\section{La règle seule : respecter les nuances}

Observons tout d'abord qu'un des aspects les plus délicats de la traduction - en particulier lorsqu'on débute - est l'intensité : comment traduire la gradation d'un phénomène ? Bien souvent, on n'en dira pas autant que le texte original - timidité, manque d'assurance, manque d'habitude - et l'on va sous-traduire : il y aura défaut d'extension entre le texte initial et sa traduction. Un exemple, tiré d'un article récent sur les logiciels de jeu d'échec et rappelant la victoire de Deep Blue sur Garry Kasparov, en 1997 :

(2) Ladmiral, 1998, p. 139.

(3) http://formations-pro.eila.univ-paris-diderot.fr/masterpro/ilts/start 


\begin{tabular}{|l|l|}
\hline Texte initial & Traduction publiée \\
\hline What could be gleaned & Quelles leçons tirer \\
from this shocking upset? & de cette déconvenue? \\
\hline
\end{tabular}

Cette traduction, ouvre collective, relue et validée par le magazine Technology Review, qui l'a publiée, est, malgré ce contrôle-qualité, un peu faible : les traducteurs se sont bien posé la question du rapport entre shocking et upset, et ont rassemblé ces deux mots, qui sinon, auraient semblé redondants, mais sans tenir compte du fait que le premier fonctionne comme un intensificateur du second. Une traduction respectueuse de la nuance aurait été, par exemple :

\section{Traduction nuancée proposée}

\section{Que nous enseigne ce bouleversement?}

Tout cela est banal. Et d'ailleurs, les sous-traductions ont ceci de confortable que, si l'on ne dispose pas du texte de départ, elles passent inaperçues. Malheureusement, elles ont aussi pour effet de banaliser le texte d'arrivée, qui perd en intensité, et donc en intérêt.

Beaucoup plus passionnante, quoique moins fréquente, est la surtraduction. Un deuxième exemple, tiré de l'histoire économique récente. Un économiste connu pour ses positions libérales écrit en 1992 une étude claironnant "The positive outcome of globalization will be huge ". Traduction française du communiqué relatif à cette publication : "Les effets positifs de la mondialisation seront colossaux."

Ici, l'échelle graduée du texte d'arrivée monte plus haut que celle du texte initial. La traduction en dit plus que l'original. Au risque de déséquilibrer l'argumentation, et donc de prêter le flanc à la critique. Et celle-ci n'a pas manqué de s'emparer de ce colossal adjectif pour attaquer, non pas la qualité de la traduction, mais directement les idées de l'auteur, en postulant - ou en voulant croire - que la formulation française était exacte. Gros problème de communication à la clef... 
Je ne surprendrai donc pas le lecteur en affirmant qu'il faut s'attacher à rendre les nuances avec un maximum de précision, en se gardant des surtraductions - ce qui est relativement aisé - comme des soustraductions - ce qui demande nettement plus de discipline. Armés de notre règle, il nous faut être rigoureux. On pourra alors reprendre la traduction suivante, extraite d'un texte sur la simulation multiéchelle dans la fabrication des puces électroniques :

\begin{tabular}{|l|l|}
\hline $\begin{array}{l}\text { A challenge for constructing pre- } \\
\text { dictive models for such }\end{array}$ & $\begin{array}{l}\text { Le plus difficile, lors de } \\
\text { systems is how to excite the dyna- }\end{array}$ \\
dictifs destinés à ces systèmes, \\
mics during model identification \\
experiments when so few mani- \\
pulated variables are available.
\end{tabular}

En effet,

- le plus difficile est trop précis par rapport à a challenge,

- parvenir est implicitement contenu dans mettre en ouvre, pour traduire how,

- malgré la rareté... rend mal l'idée que cette rareté fait partie des conditions de départ de l'exercice. Mieux vaudrait, en première approximation, inverser l'ordre :

Compte tenu de la rareté des variables manipulées, une des difficultés rencontrées dans l'élaboration des modèles prédictifs destinés à ces systèmes consiste à mettre en œuvre une simulation dynamique pendant les phases d'identification de modèle.

Plus hardiment encore, on pourra décider de transformer notre apposition en sujet, sous réserve d'avoir vérifié (auprès d'un expert) qu'on 80 ne disait pas de bêtise : 
La rareté des variables manipulées dans l'élaboration des modèles prédictifs destinés à ces systèmes complique la mise en œuvre d'une simulation dynamique pendant les phases d'identification de modèle.

Juste une remarque incidente : ce qui a amené notre traducteur à être imprécis sur les nuances, c'est une préoccupation - aussi légitime que respectable - pour la terminologie. Il s'est en effet concentré sur les (réels) problèmes que posent les mots " excite the dynamics " et "model identification experiments", ce qui lui a fait perdre de vue le reste de la phrase. C'est fondamental, la terminologie - j'y reviendrai dans peu de paragraphes -, mais ce n'est pas tout.

Revenons donc à nos nuances. Nous commençons à peine à entrevoir le problème. Finalement, avec une règle, on ne fait pas grand chose. Car la pratique nous apprend qu'il existe des contre-exemples. Et c'est par les contre-exemples qu'on peut arriver à donner une représentation honnête des réalités. C'est là que nous compléterons notre premier instrument par un second : le compas.

\section{La règle et le compas : faire varier la forme}

Notre traducteur doit en effet aussi apprendre à faire varier, non pas la graduation, mais l'extension du domaine couvert par sa traduction. Dans sa recherche de la précision et de l'exactitude, il va se heurter, presque toujours, à la question du choix : tout cela est évident et bien connu ; inutile de s'y attarder. Là où il va devoir choisir en géomètre, c'est face à certaines formulations qui l'obligeront à écarter ou à resserrer les pointes de son compas.

Le cas le plus évident est bien sûr la gestion de la redondance et celle de l'implicite : nous jouerons ici sur la forme.

Ainsi, dans l'exemple suivant - ironiquement tiré d'une publicité vantant, en anglais et en français, les mérites d'une agence de traduction aujourd'hui disparue -, il n'y a aucun doute possible sur le ridicule qui provient des redondances en français. 
Every member of our translation and localization teams is a trained subject expert with at least a decade of proven performance in the translation industry.

Tous les membres de nos équipes de traduction et de localisation sont des experts ayant été formés dans leur domaine de spécialité et pouvant justifier d'au moins dix ans d'expérience dans le domaine de la traduction.

Rappelons que, en dehors de la trilogie Matrix, on ne peut pas être expert sans avoir reçu une formation, fût-ce sur le tas, et que lorsqu'on est expert, c'est généralement parce qu'on possède une spécialisation... Pour traiter ces redondances, il faut apprendre à raisonner par l'absurde, c'est-à-dire à inverser les formulations pour voir si le résultat a encore un sens. Si c'est le cas, alors, les mots concernés ont bel et bien une utilité. Ici, cela donnerait :

Tous les membres de nos équipes de traduction et de localisation sont des experts qui n'ont reçu aucune formation et ne possèdent aucun domaine de spécialité [...].

Éliminer les lourdeurs de ce genre ne demande donc pas un degré de sophistication intellectuelle très poussé : nous sommes encore dans le registre de la banalité. Sans être follement originale, la gestion de l'implicite est déjà un peu plus subtile. Elle consiste à faire ressortir des éléments de sens qui peuvent être absents ou discrets dans le texte de départ. Ce sera notre nouvel exemple, tiré d'un texte sur l'utilisation de puces radiofréquences (ou tags RFID) dans la logistique d'une maison de disques, afin de pouvoir suivre à la trace chaque $\mathrm{CD}$ de 82 l'usine jusqu'à la caisse enregistreuse. 


\section{Précision (utilement) ajoutée}

The system flagged a discrepancy in the first shipment [...], although the missing $C D$ was later found and scanned into the system to complete the consignment. This highlighted the value of visible transactional data.
Le système a signalé un écart dans la première livraison [...], mais le $\mathrm{CD}$ manquant a ensuite été retrouvé et réintégré dans le système afin de compléter la livraison. Cet incident a mis en valeur l'intérêt d'une visibilité des données concernant les mouvements.

L'amplification du this me paraît une excellente idée. Il faut en effet chercher toujours à être le plus précis, en remontant dans l'ordre des catégories : de quel type d'événement ou de situation s'agit-il, comment qualifier ce qui arrive ? La traduction, ne l'oublions jamais, passe par un acte de nomination. C'est son héritage judéo-chrétien, et c'est pour cela qu'une bonne traduction suppose la mise en place d'une bonne terminologie, c'est-à-dire, en pratique, la constitution d'une ontologie, qui permette de reconstituer l'univers dans lequel évolue le texte à traduire.

Cette gestion de l'implicite va aussi, très souvent, nous amener à jouer sur l'insistance. Que fait-on, quand on traduit, finalement, de manière très concrète ? On détermine, dans un passage, les éléments qui sont porteurs de sens, et on s'en sert pour recomposer un passage équivalent dans la langue d'arrivée (processus qui n'a rien de linéaire). Par exemple, dans un texte sur le contrôle des armes légères dans les pays menacés par la guerre civile, je peux être confronté à la phrase suivante :

Insistance, texte original

Most demand reduction occurs at the local level. 
Or, tout mon texte traite des moyens de réduire la demande. Ce n'est donc pas là-dessus que je dois insister : l'élément nouveau, le point réellement pertinent, c'est l'adjectif local :

\section{Insistance, version française}

C'est le plus souvent à l'échelon local que l'on parvient à réduire la demande d'armes.

Autre exemple, provenant d'un texte américain sur le lien entre la nature des régimes d'actionnariat des salariés (ESOP, dans notre texte) et le degré de protection de l'entreprise considérée contre les offres publiques d'achat (OPA) :

Insistance, bis

Chaplinsky and Niehaus (1990) Chaplinsky et Niehaus (1990) find that ESOPs are not mana- montrent que la gestion des ged to provide the maximum tax ESOP ne permet pas d'assurer benefits to shareholders. les meilleurs avantages fiscaux aux actionnaires.

Sans être véritablement fausse, cette traduction passe à côté du problème, qui, en contexte, consiste à savoir si les régimes d'actionnariat ne sont pas un moyen déguisé de se prémunir contre les OPA hostiles. Il faudrait donc recentrer la traduction sur cette préoccupation, pour lui faire dire :

Chaplinsky et Niehaus (1990) montrent que les ESOP ne sont pas gérés prioritairement en vue d'assurer les meilleurs avantages fiscaux aux actionnaires.

Nous restons toutefois, ici, dans le registre de l'exactitude, dans un mode de pensée symbolisable par la règle. L'usage du compas, c'est-àdire la variation de l'amplitude dans l'expression, se limite encore à l'emploi de moyens rhétoriques. Cela passe par un jeu d'ajouts, de 
suppressions et d'insistances qui montre bien que la traduction est avant tout un art différentiel. C'est un des points sur lesquels la traduction automatique - qui a par ailleurs ses mérites et son intérêt - ne peut qu'achopper(4). Mais nous ne sommes encore nulle part intervenus sur le sens : la situation demeure donc simple.

\section{Raisonner juste sur des figures fausses}

Pourtant, dans certains cas, je vais me sentir obligé de modifier véritablement une nuance, c'est-à-dire d'intervenir sur le fond, sur le sens même du texte. Je vais donc procéder à des projections géométriques, dans un premier cas pour restituer non plus ce que le texte dit, mais ce qu'il fait, dans un deuxième, pour hiérarchiser les susceptibilités en fonction de l'intention que je prête à ma traduction et, dans un troisième, pour montrer que l'entre-deux n'est pas un lieu qui convient aux traducteurs.

\section{Traduire ce que le texte fait}

Ce qui est intéressant, dans le premier cas, c'est que les écarts par rapport à l'original ont tendance, avec le métier, à jaillir spontanément sous la plume. Ils sont judicieux (généralement), mais pas forcément faciles à justifier. Un nouvel exemple, provenant d'un article scientifique sur le rôle de la modélisation en rapport avec les nanotechnologies :

Heureux changement de nuance (mais comment le justifier ?)

This trend of the semiconductor industry towards multiscale simulation began to be explored a decade ago, and has been an active area of research [...].
Cette évolution de la profession vers la simulation multiéchelle a démarré il y une dizaine d'années et la recherche dans ce domaine est, depuis lors, très active $[\ldots]$.

(4) Voir Froeliger, 2007. 
La difficulté, une fois que l'ajout de ce très se retrouve sur la page ou à l'écran, consiste à le rationaliser, à le justifier. Après réflexion et discussion, on peut penser que c'est parce que la recherche est active $a$ priori. Si l'on veut que l'anglais active soit porteur de sens dans le texte français, il faut alors ajouter un intensificateur. (On pourra aussi décider de noyer le poisson, en utilisant l'adjectif bouillonnant, ou en déplaçant l'adjectif, par exemple.)

D'autres changements de nuance sont nettement plus faciles à justifier. Ainsi l'exemple suivant :

\section{Problème d'exactitude}

Some RF tags are now smaller Il existe aujourd'hui des tags than grains of rice! RFID de la taille d'un grain de riz!

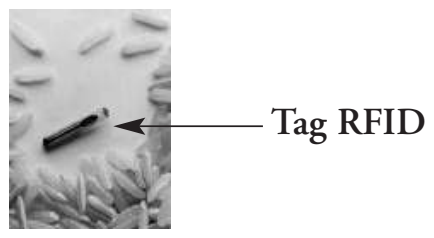

Si l'on ne regarde que les mots, le traducteur est dans l'erreur. En fait, il a donné la préférence à l'image par rapport à sa légende, et a donc correctement rectifié une inexactitude de l'original. La solution la plus prudente, pour ménager chèvre et chou, aurait voulu : Il existe aujourd'hui des tags RFID de la taille d'un grain de riz, voire encore plus petits ! Mais c'est aussi beaucoup plus lourd, et donc moins efficace.

Comment justifier ces deux écarts volontaires par rapport au sens apparent du texte de départ ? Tout simplement par l'existence du monde : les textes pragmatiques ont une visée de communication et sont référés au monde. En d'autres termes, ils ne sont pas tout entiers contenus dans leur formulation linguistique. Si c'était le cas, on pourrait se contenter d'en restituer le sens apparent avec la plus grande 
exactitude, mais l'obligation de nous raccorder au réel fait que ce que nous devons véritablement traduire, c'est l'intention que nous prêtons au texte dans l'usage concret qui doit en être fait. Le réel, c'est la photographie des grains de riz à côté de notre puce radiofréquence, c'est la nature intrinsèquement active de la recherche. Donc, lorsqu'on traduit, on n'est pas dans une relation bilatérale entre texte de départ et texte d'arrivée, mais dans un rapport triangulaire (voire quadrangulaire, lorsque le référent n'est pas le même pour les deux langues) qui inclut le réel comme variable de contrôle. En traduisant non pas les mots que nous avons sous les yeux, mais ce que notre texte est censé faire dans le monde réel, nous restons, ici, géomètres.

\section{Ménager les susceptibilités}

Et la géométrie, c'est notamment (rappelons-le) la traduction en phénomènes sensibles de relations mathématiques. On doit donc traduire avec sensibilité, par exemple face à une formulation qui pourra sembler offensante :

Die $\mathrm{CO}_{2}$-Emissionen des Verkehrssektors in Deutschland haben sich auf Basis der IEA-Daten geringer gesteigert als in allen anderen wichtigen EU-Ländern.

Par respect pour les Maltais, Slovaques, Lettons et autres Slovènes, je vais me sentir obligé d'atténuer légèrement ce wichtigen (littéralement, "les pays européens importants", ce qui sous-entend que les autres ne le sont pas). Pourquoi cette atténuation ? Parce que c'est utile pour la visée de mon texte :

Selon les statistiques de l'Agence internationale de l'énergie (AIE), les émissions de $\mathrm{CO}_{2}$ liées aux transports ont augmenté moins fortement en Allemagne que dans tous les autres grands pays de l'Union européenne.

Ici, lorsque je change de métaphore de référence, je dois être sûr que le périmètre couvert est le même : nous sommes passés d'une question 
de poids économique et politique à une simple extension géographique. Pour m'assurer que cela fonctionne, je dois réfléchir à l'existence éventuelle d'exceptions : là encore, nous raisonnons par le contre-exemple.

Cependant, l'opération est délicate : ma traduction flirte avec le politiquement correct, qui ne donne pas souvent de formulations convaincantes et, en pratique, je sous-traduis, après avoir dit tant de mal de la sous-traduction. Il y aurait beaucoup à dire sur la nécessité et les excès de la diplomatie dans ce métier...

Cela nous amène, en attendant, à un nouvel exemple, source d'un deuxième incident diplomatique véritable, dû à une traduction peutêtre un peu trop naïve dans son respect de la nuance. Dans un texte sur les perspectives économiques dans différents pays africains, on trouvait, en 2005, la phrase suivante :

It would appear that President Wade's honeymoon with the people is coming to an end.

Aucun problème de traduction, a priori :

Il semble que la lune de miel entre le président Wade et sa population touche à sa fin.

C'est effectivement ainsi que les traducteurs ont procédé. Et déclenché une grosse fâcherie entre l'organisme ayant publié cette phrase et le Sénégal. Car la fin d'une lune de miel, c'est la disparition de l'amour. Ce que l'auteur, consulté après coup, voulait dire, c'est que la période où l'on pardonne tout à un dirigeant nouvellement arrivé au pouvoir était en train de se terminer. Cliché pour cliché, il aurait fallu écrire :

C'est semble-t-il la fin de l'état de grâce pour le président Wade.

Respecter l'intention, ici, c'est changer d'expression et, au passage, faire disparaître le peuple... Là où nous nous sommes, tout à l'heure, aidés du réel pour représenter l'intention du texte en contexte, nous 
procédons ici à une autre forme de triangulation : il s'agit, un peu comme chez Platon, de projeter une représentation idéelle du texte et de traduire celle-ci, plutôt que la formulation imparfaite qu'on a sous les yeux, ou tout au moins sa capacité à vouloir dire autre chose que ce qu'il semble dire en surface. À l'instigation de René Descartes, nous raisonnons donc juste sur des figures fausses.

\section{La géométrie a horreur de l'entre-deux}

Notre dernier cas de figure est sans doute le plus subtil. Nous avons vu que la traduction consistait, entre autres choses, à jouer sur les ajouts et les suppressions en fonction des éléments porteurs de sens et de leur poids relatif. Mais que faire lorsque je ne suis pas certain que tel élément, tel mot, veut réellement dire quelque chose dans mon texte de départ ? Encore un exemple, concernant les politiques de lutte contre les discriminations dont sont victimes les populations roms :

Governments should take positive steps to protect housing rights of Roma in Europe, UN and Council of Europe experts say.

Ladjectif positive sert-il, ici, à quelque chose ? Si j'emprunte l'outil du raisonnement par l'absurde, je conclurai forcément par la négative : peut-on conseiller à une administration publique de prendre des mesures négatives... ? Donc, j'aurai tendance à omettre ce mot de ma traduction :

Des experts des Nations unies et du Conseil de l'Europe recommandent l'adoption de mesures pour protéger le droit au logement des Roms en Europe.

Mais je n'ai pas la certitude que ce mot soit purement décoratif en anglais. Après tout, il a peut-être été placé dans mon texte parce que certaines mesures adoptées par le passé n'étaient que poudre aux yeux, effets d'annonce et autres fumigènes, comme on dit depuis quelques mois au Palais de l'Élysée... Et dans ce cas, l'élision serait une grave faute. Il faudrait au contraire insister : 
Des experts des Nations unies et du Conseil de l'Europe recommandent l'adoption de mesures réellement efficaces/concrètes/ constructives/volontaristes pour protéger le droit au logement des Roms en Europe(5).

Nous retrouvons ici notre premier exemple. Après tout, j'aurais aussi pu choisir de traduire les deux mots shocking upset par deux mots français, au lieu de rassembler en une seule unité de sens :

\begin{tabular}{|l|l|}
\hline Texte initial & Traduction publiée \\
\hline $\begin{array}{l}\text { What could be gleaned from } \\
\text { this shocking upset? }\end{array}$ & $\begin{array}{l}\text { Quelles leçons tirer de cette ter- } \\
\text { rible déconvenue? }\end{array}$ \\
\hline
\end{tabular}

Mais terrible est bien plus fort que shocking...

Face à un mot qui me gêne dans l'original, j'ai donc finalement le choix entre l'élision - je supprime en partant du principe que l'équivalence est conservée - ou l'ajout - je survalorise. Seul le moyen terme, ici, m'est interdit : traduire, cela reste choisir. En tout cas à mes yeux. Est-ce que le sens est le même ? Est-ce que procéder ainsi, c'est déverbaliser? Je ne sais pas. Et c'est parce que je ne sais pas qu'il m'a paru souhaitable de dialoguer avec des professionnels sur ce point. Ce que je sais, en revanche, avec certitude, c'est que cette question revient avec insistance lorsqu'on traduit. Alors, sur quels critères vais-je trancher entre le vide et le trop-plein ? En fonction, là encore, de l'intention précise que je prête à mon texte dans le cadre d'une demande elle aussi précise : je dois adopter une posture, qui va déterminer le degré d'ouverture de mon compas.

(5) La discussion de cet exemple avec les participants à la Journée de la Traduction Professionnelle 2008 de la SFT où il a été présenté montre que les préférences sont variables : certains privilégient la suppression, d'autres l'insistance, d'autres, encore, la prudence ("mesures positives "), qui a, à mes yeux, pour effet de laisser au destinataire le choix de l'interprétation en signalant par ce non-choix que l'on a affaire à un texte traduit et non plus à un écrit ayant vocation d'original : et dieu reconnaîtra les siens... 
Tout le problème est donc dans la netteté de la perception - celle de l'auteur, la mienne, celle de mon ou mes lecteurs : comprendre, réexprimer. Mais le traitement de la nuance nous conduit, à l'occasion, à admettre - et même à postuler - l'imperfection du texte de départ. Or, cette opération est délicate : qui suis-je pour affirmer que je saurai mieux que mon auteur comment dire ce qu'il a voulu dire ? On court, ici, le risque de l'interprétation, qui est une forme de démesure. Avant d'intervenir sur la substance du texte, il faut donc savoir être rigoureux : on ne s'écarte de la tonalité d'origine qu'à bon escient. On apprend à manier la règle avant le compas. Toute la difficulté d'une traduction nuancée consiste donc à savoir se détacher de la formulation de départ tout en serrant l'intention au plus près et en sachant à quel moment la situation de communication demande une manipulation de la nuance.

Néanmoins, arrivé à ce stade - tardif - de ma démonstration, je suis pris de doute : finalement, ces questions de nuance présentent-elles un réel intérêt, et pour qui ? Il est bien évident que la qualité ne se décrète pas; elle se construit. On est alors tenté de poser une question : la recherche forcenée de la nuance appropriée est-elle toujours un gage de qualité, ou bien peut-elle nuire à celle-ci ? Quelle est la pratique ? Les sondages empiriques auprès d'un large panel de professionnels donnent des résultats contrastés ; certains traducteurs privilégieront la fluidité, d'autres s'évertueront à restituer la moindre finesse, au risque paradoxal de la lourdeur... Cette diversité montre bien que maints problèmes de nuance - je pense même la majorité - passeront parfaitement inaperçus et que leur consacrer trop d'attention pourra se révéler contre-productif : si on regarde un texte - n'importe lequel - de trop près, tout devient énigmatique... Est-il, dans ces conditions, souhaitable de nuancer l'importance de la nuance ? Je ne crois pas. En effet, il est difficile, même en rassemblant toutes les informations nécessaires sur la nature du travail qu'on attend de nous, d'évaluer à quel endroit elle sera décisive et à quel autre elle est indifférente, ce qui nous oblige finalement à être maniaques, obsédés et psychorigides en toute occasion dans nos traductions (et là seulement, espérons-le), 
sans savoir si c'est réellement utile et raisonnable. C'est donc d'abord une forme résolue de prudence qui nous incite à la nuance. Mais pas seulement. Car même si elle n'a aucune importance pour nos clients, demandeurs et autres destinataires, cette forme de respect en revêt une pour nous-mêmes. Avant d'être déontologique, le ressort de cette préoccupation est donc, je pense, éthique.

\section{Remerciements :}

Christine Durban, Architexte, Colm Foy, Agnès Gallais-Belaïd, Andrea Incarbona, Fabien Moll-François, Françoise Wirth, Nathalie Renevier et bien d'autres.

nf@eila.univ-paris-diderot.fr

Diplômé de l'ESIT et auteur d'une thèse sur Thomas Pynchon, Nicolas Froeliger a été traducteur technique au sein de la société Architexte. Il est aujourd'hui maître de conférences à l'Université Paris VII, où il enseigne l'anglais et la traduction de textes pragmatiques et est responsable du Master professionnel ILTS (Industrie de la langue et traduction spécialisée). Ses travaux antérieurs ont porté sur le cliché, l'interdisciplinarité, la forme en traduction technique, la question des personnages en traduction de textes pragmatiques et les postures nécessaires à l'exercice de la traduction. Il s'intéresse globalement aux passerelles susceptibles d'exister entre savoir technique, culture générale et culture littéraire.

\section{Références}

Froeliger, Nicolas, 2007, Pourquoi les traducteurs craignent-ils l'informatisation de leur profession? TILV (Tribune internationale des langues vivantes), $n^{\circ} 43$.

Ladmiral, Jean-René, 1998, "Théorie de la traduction : la question du littéralisme ", extrait du colloque Théorie et pratique, organisé par l'Institut catholique de Paris, paru dans Transversalités, janvier-mars, $92 \quad$ pp. 137-157. 
Steiner, George, 1975, After Babel, Aspects of Language and Translation, Oxford University Press, New York et Londres, 1975. Nous nous référons à la traduction française de Lucienne Lotringer, Après Babel, Albin Michel, 1978.

Warusfel, André, 1961, Les nombres et leurs mystères, Éditions du Seuil, Paris. Nous nous référons à l'édition Points Seuil, 1980, p. 85. 